\title{
Efeito da Presença de Vizinhos sobre o Comportamento Territorial de Perithemis mooma (Kirby) (Anisoptera: Libellulidae)
}

\author{
Nelson Silva Pinto1 ${ }^{\bowtie}$, José Hidasi Neto ${ }^{1}$, Vivian Ribeiro², Amanda Rafaela Rodrigues, \\ Bárbara Regina Brandão ${ }^{1}$ \& Camila Oliveira Rocha ${ }^{1}$
}

1. Universidade Federal de Goiás, e-mail: nelsonsilvapinto@gmail.com (Autor para correspondência ${ }^{\bowtie}$ ), hidasineto@gmail.com, amandaraphaella@yahoo.com.br, barbarareginabebe@hotmail.com, camila ufg2006@hotmail.com. 2. Universidade de Brasília, e-mail: lacutaco@hotmail.com.

\section{EntomoBrasilis 6(2): 104-107 (2013)}

Resumo. Algumas espécies territorialistas apresentam respostas menos agressivas em relação à entrada de vizinhos do que a não vizinhos em seu território. Essa diferenciação nas respostas é conhecida como "Dear Enemy Effect". O objetivo deste estudo foi avaliar as interações de Perithemis mooma (Kirby) com seus vizinhos e invasores, testando a ocorrência do "Dear Enemy Effect". Este estudo foi conduzido no Campus Samambaia da Universidade Federal de Goiás (UFG). As interações agonísticas intraespecíficas de $P$. mooma foram observadas em 30 indivíduos, totalizando 300 minutos de observação. Verificamos que os indivíduos passam mais tempo nas brigas com invasores. Essa informação corrobora a hipótese de que os indivíduos reconhecem os vizinhos, consequentemente gastando menos tempo nas interações agonísticas com eles. Para a análise sobre o tempo e proporção de briga com vizinhos mais e menos distantes, verificamos que os indivíduos passam mais tempo em interações com vizinhos mais próximos do que com vizinhos mais distantes. Ocorreu maior proporção de briga com invasores provenientes dos lados dos vizinhos menos distantes do que com os provenientes do centro (teste de Tukey; $\mathrm{p}=0,034$ ). Observou-se que em $P$. mooma existe um efeito significativo indicando que machos que se reconhecem como vizinhos interagem com menor agressividade do que quando interagem com invasores. Desta forma, é possível sugerir que existe efeito "dear enemy" em machos de P. mooma, visto que houve diferença significativa no tempo, bem como na intensidade das interações com vizinhos e com invasores.

Palavras-chave: Efeito do Inimigo Próximo; Interações agonísticas; Sistema de acasalamento; Territorialidade.

\section{Effect of the Presence of Neighbors on the Behavior of Territorial Perithemis mooma (Kirby) (Anisoptera: Odonata)}

Abstract. Some territorial species present less aggressive responses in relation to the entering of neighbors than non-neighbors in their territory. This differentiation in responses is known as "Dear Enemy Effect". The objective of this study was evaluate the interaction of Perithemis mooma (Kirby) with its neighbors and invaders, testing the occurrence of the "Dear Enemy Effect". This study was conducted in the Campus Samambaia of the Universidade Federal de Goiás (UFG). The intraspecific agonistic interactions of $P$. mooma were observed in 30 individuals, totaling 300 minutes of observation. We verified that the individuals spend more time in fights with invaders. This information corroborates the hypothesis that individuals recognize the neighbors; consequently spend less time the agonistic interactions with them. For the analysis about time and proportion of fight with neighbors more or less distant, we verified that the individuals spend more time in interactions with closer neighbor than further neighbors. Occurred more proportion of fight with invaders from the side of the lesser distant than from the center (Tukey test; $\mathrm{p}=0.034$ ). We observed that in $P$. mooma there is a significant effect indicating that males who recognizes themselves as neighbors interact with lesser aggressively than when interact with invaders. Thus, it is possible suggest that there is an effect dear enemy in males $P$. mooma, since there was a significant difference in time, as well as in the intensity of the interaction with neighbors and with invaders.

Keywords: Agonist Interactions; Dear Enemy Effect; Mating System; Territoriality.

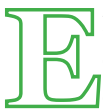
$\mathrm{m}$ alguns gêneros de Odonata o sucesso de reprodução está relacionado à capacidade dos machos em defender os territórios e controlar o acesso das fêmeas aos recursos necessários para a postura dos ovos (CORBET 1999). As libélulas do gênero Perithemis são territoriais e defendem seu território de forma agressiva para afastar outros machos ou fazer captura em voo (Switzer 2002). A preferência dos machos por áreas com vegetação para os territórios deve estar relacionada com a seleção local de oviposição pelas fêmeas, o que conduz à agregação de vários machos em torno dos locais com maiores agregações dos recursos utilizados pelas fêmeas (DE MARCO \& RESENDE 2004). Disputas pela manutenção de territórios e, consequentemente, de potenciais parceiros para reprodução, pode culminar em comportamentos agonísticos entre indivíduos de uma mesma espécie (Switzer 2002).

Entretanto, devido possivelmente ao reconhecimento entre 'vizinhos' (indivíduos que dividem territórios ou dominam territórios contíguos), alguns indivíduos respondem de forma menos agressiva à entrada de seus vizinhos do que a entrada de não vizinhos em seu território. Essa diferenciação nas respostas entre vizinhos é conhecida como "Dear Enemy Effect" (SwITZER \& EASON 2003). Uma explicação para esse fenômeno se baseia na familiaridade (reconhecimento entre os indivíduos ao longo do tempo, devido a repetidas interações entre esses) entre vizinhos, uma vez que as fronteiras do território estão estabelecidas, os vizinhos representam pouca ou nenhuma ameaça, enquanto um indivíduo de território distante corresponde a um estranho que pode estar em busca de um novo território (Temeles 1994), podese também considerar o menor custo energético de uma interação agressiva prevenindo lutas com vizinhos que já são frequentes. 
T Alguns pesquisadores têm considerado que a presença de vizinhos é benéfica a algumas espécies territorialistas, principalmente em comparação com competidores não vizinhos da mesma espécie, no entanto, os vizinhos podem representar custos tanto em termos de defesa de território quando de recursos perdidos (Switzer \& EASON 2003). A espécie Perithemis mooma (Kirby) é um espécie de Odonata associada à defesa de territórios e é típica de ambientes lênticos do tipo lacustre (GARRISON et al. 2006). Por ser uma espécie territorialista, geralmente é feito o reconhecimento dos vizinhos a fim de reduzir os custos energéticos de interações para disputa de territórios e parceiros para cópula.

Esta espécie de Odonata é tipicamente associada a ambientes lênticos do tipo lacustre (GARRISON et al. 2006), defendendo territórios em áreas com vegetação, muito embora não seja afetada pelo tamanho do poleiro, ou seja, pela altura da vegetação no entorno (De Marco JR \& Resende 2004) o que nos permite utilizar os poleiros naturais, sem necessidade de manipulação. Com alta incidência em locais com vegetação escassa e devido à conspicuidade da espécie, os indivíduos de $P$. mooma foram selecionados para que fossem feitas análises relativas a eventos de territorialismo, objetivando o estudo das interações destes indivíduos com seus vizinhos e com invasores não vizinhos, a fim de testar a ocorrência do "Dear Enemy Effect".

\section{MATERIAL E MÉTODOS}

Área de estudo. Este estudo foi conduzido em tanques de piscicultura, localizados na Escola de Agronomia e Engenharia de Alimentos, Campus Samambaia da Universidade Federal de Goiás (UFG), município de Goiânia ( $\left(6^{\circ} 40^{\prime}\right.$ S, $49^{\circ} 15^{\prime}$ O, alt. 749 $\mathrm{m})$. Os tanques são tanques-terra construídos para abrigar peixes para pesquisa e comércio. Estes tanques apresentam vegetação escassa, consistindo principalmente de gramíneas. Os indivíduos do gênero Perithemis utilizam essa vegetação como poleiros onde defendem territórios de aproximadamente $2,5 \mathrm{~m}$ de raio (SwITZER 2002), no qual respondem agonisticamente a machos intrusos.

Método de amostragem. A amostragem foi conduzida apenas quando a temperatura esteve acima de $19^{\circ} \mathrm{C}$, e entre as 11:00 e 15:0oh, pois fora destes parâmetros a atividade de Odonata decresce muito (MAY 1976; DE MARCO JR \& RESENDE 2004). O método de levantamento dos indivíduos foi baseado no método de varredura por scan (DE MARCo 1998), muito utilizado em estudos com estes organismos (PinTo 2011; DE MARCo 2008). Foram analisados machos que apresentavam dois vizinhos, sendo que ambos (vizinho-esquerda e vizinho-direita) deveriam estar dentro do território do macho focal/residente (SWITZER 2002). Quatro pesquisadores observaram ao mesmo tempo os indivíduos por um período de 10 minutos de amostragem visual, sendo que três desses observadores analisaram as interações dos machos residentes com seus vizinhos e com invasores (machos nãoterritoriais que tentam invadir o território), que poderiam chegar para uma interação com o macho focal pelo lado do vizinho mais próximo ou pelo lado do vizinho mais distante. As proporções dessas interações foram transformadas em arco-seno da raiz, para que os dados fossem normalizados (ZAR 1999). O quarto observador anotou os voos realizados pelo macho residente, bem como para onde estes voos se destinaram. Estes voos poderiam ser contra os vizinhos ou intrusos. Além disso, o terceiro observador também mediu o tempo, em segundos, das interações de briga entre os vizinhos e os invasores. Com o intuito de verificar se de fato houve intrusão ao território do macho observado, seguimos a sugestão de SwITZER \& EASON (2003), de que os territórios das espécies de Perithemis têm aproximadamente $2,5 \mathrm{~m}$ de raio.

As interações agonísticas intraespecíficas de $P$. mooma foram observadas em 30 indivíduos, totalizando 300 minutos de observação. Destes, foram retiradas observações nos quais a disposição dos vizinhos era equidistante, para que fosse possível verificar se havia diferenças significativas entre vizinhos mais próximos e mais distantes.
Análise estatística. Para testar se ocorreu maior tempo de interação agonística dos residentes com os invasores do que com os vizinhos, foi feito um teste-t entre os respectivos valores de tempo. Em seguida, um segundo teste-t foi feito para que se observasse se ocorre maior tempo de briga com vizinhos mais distantes do que com vizinhos mais próximos. Um terceiro teste-t foi conduzido para observar se a proporção de interações agonísticas foi maior com o vizinho mais distante do que com o mais próximo. Em seguida, foram utilizados dois testes ANOVA: para que medir se o tempo de briga com invasores do lado do vizinho mais distante era maior do que do lado do vizinho mais próximo ou do que eram provenientes do centro; e para analisar se a proporção de intrusão era maior do lado do vizinho mais distante do que do vizinho mais próximo ou proveniente do centro.

\section{RESULTADOS}

O tempo de briga com invasores é maior do que com vizinhos? Para a análise sobre o tempo de briga com vizinhos e invasores, verificou-se que os indivíduos passam mais tempo nas brigas com invasores $(\mathrm{t}=|-3,365|$; g.l. $=355$; $\mathrm{p}<0,001$; Figura 1). Essa informação corrobora a hipótese de que os indivíduos reconhecem os vizinhos, consequentemente gastando menos tempo nas interações agonísticas com eles ("Dear Enemy Effect”).

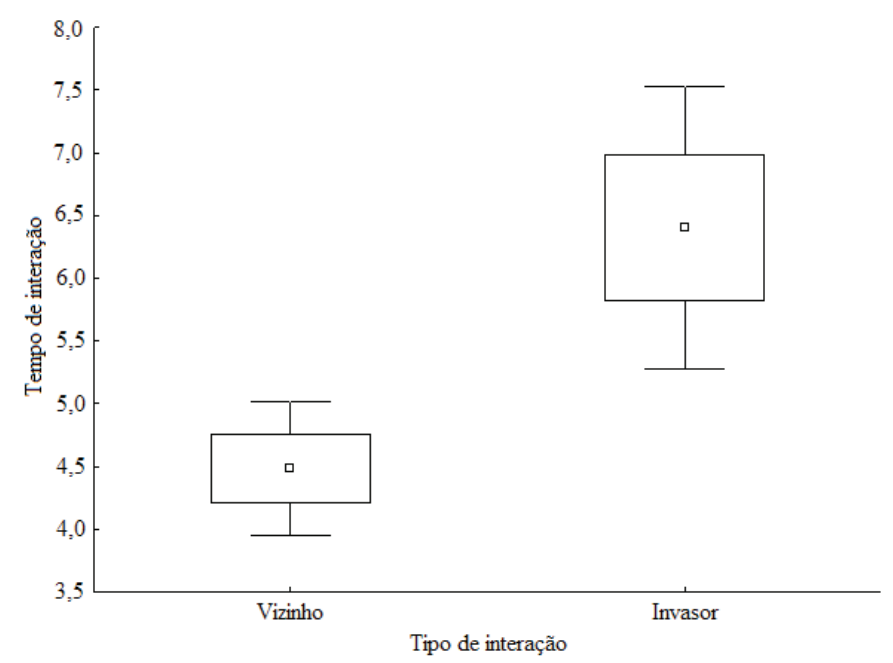

Figura 1. Tempo de interação agonística intraespecífica de Perithemis mooma com seus vizinhos e com invasores, e seus respectivos errospadrão.

O tempo e proporção de briga com vizinhos próximos são maiores do que com os distantes. Nossa hipótese de que as interações entre o macho focal/residente e o vizinho mais distantefossem mais intensas e, consequentemente, haveria maior investimento de tempo nestas interações, não foi corroborada. Para a análise sobre o tempo e proporção de briga com vizinhos mais e menos distantes, verificamos que os indivíduos passam mais tempo e brigam mais com vizinhos mais próximos do que com vizinhos mais distantes $(\mathrm{t}=2,7600$; g.l. $=197$; $\mathrm{p}<0,001$ e $\mathrm{t}=$ 2,909; g.l. $=340 ; p=0,006$; Figuras 2 e 3, respectivamente).

Diferença entre o número de invasões. Para a análise sobre o tempo de briga com invasores provenientes do centro e dos lados dos vizinhos mais e menos distantes, foi encontrado que os indivíduos não passam maior tempo de interações agonísticas nem brigam mais com invasores provenientes dos lados dos vizinhos mais distantes $[\mathrm{F}(2,68)=0,2158, \mathrm{p}=0,806$; Figura 4]. Entretanto, ocorreu maior proporção de briga com invasores provenientes dos lados dos vizinhos menos distantes do que com os provenientes do centro (teste de Tukey: $\mathrm{p}=0,034$ ). Este resultado não corrobora a nossa hipótese de que brigas com invasores seriam mais comuns dos lados dos vizinhos mais distantes, e que estes teriam um maior tempo de briga com os indivíduos observados $[\mathrm{F}(2,48)=3,392, \mathrm{p}=0,042$; Figura 5]. 


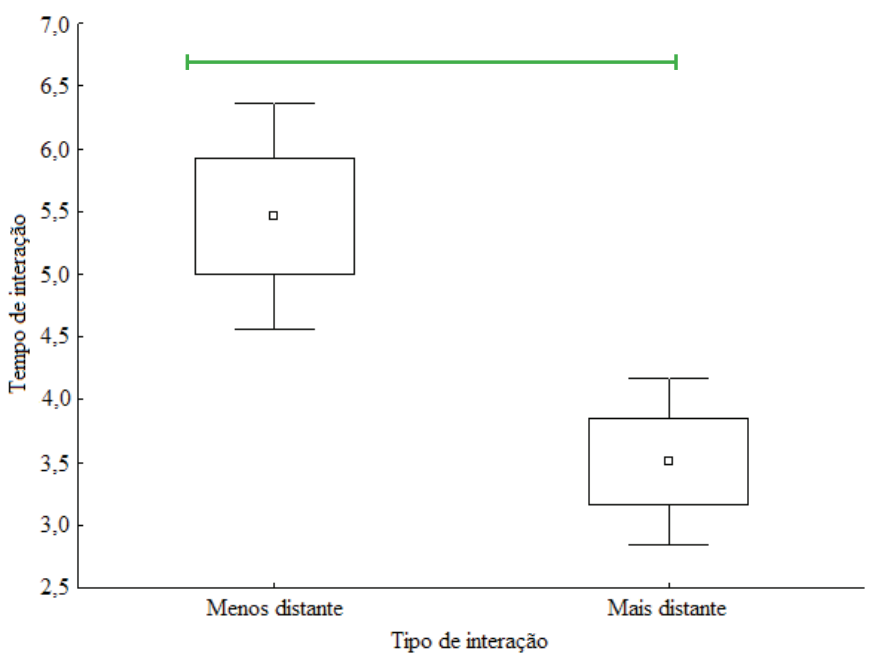

Figura 2. Tempo de interação agonística intraespecífica de Perithemis mooma com seus vizinhos menos e mais distantes, e seus respectivos erros-padrão.

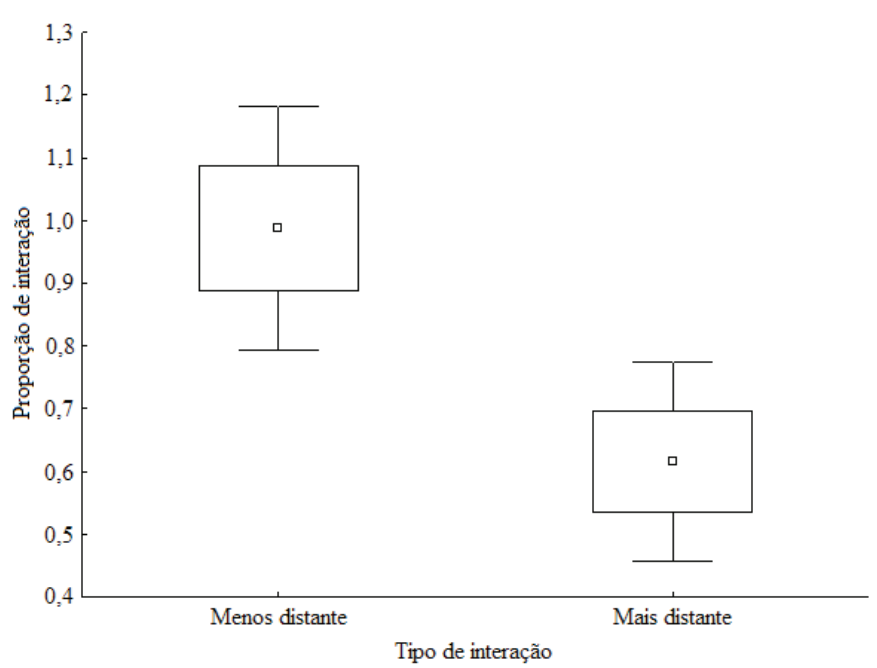

Figura 3. Proporção de interação agonística intraespecífica de Perithemis mooma com seus vizinhos menos e mais distantes, e seus respectivos erros-padrão.

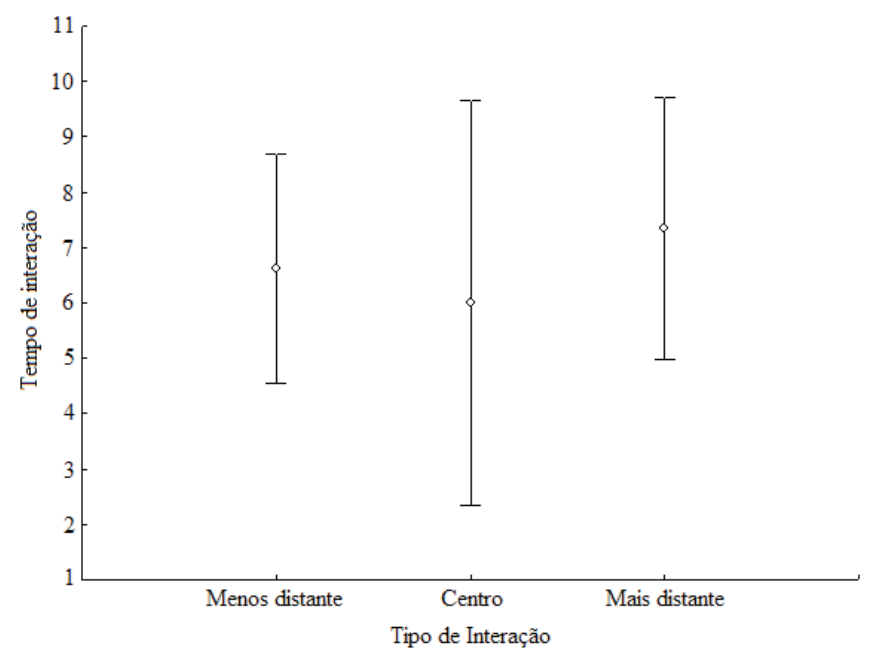

Figura 4. Tempo de interação agonística intraespecífica de Perithemis mooma com invasores provenientes do centro e dos lados dos vizinhos menos e mais distantes, e seus respectivos intervalos de confiança.

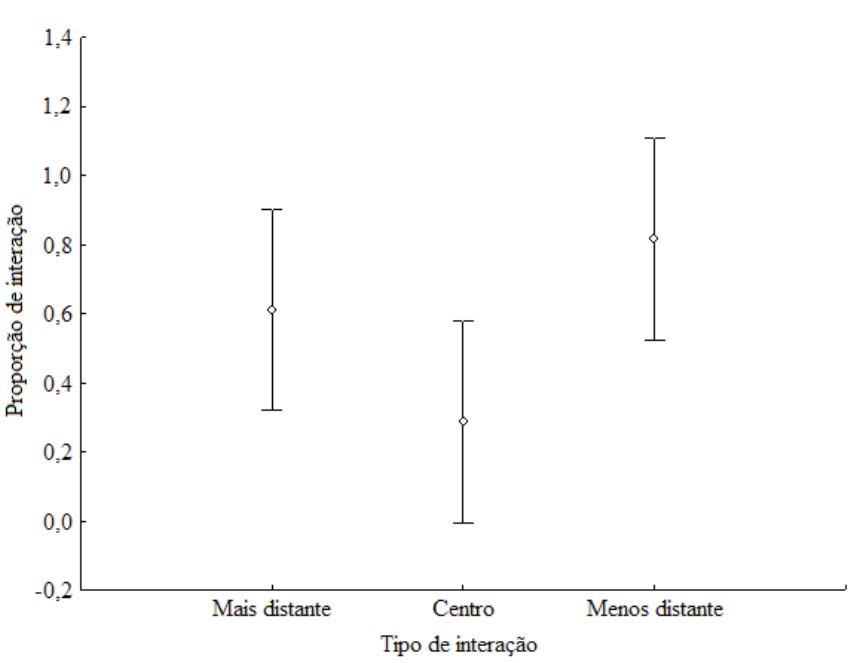

Figura 5. Proporção de interação agonística intraespecífica de Perithemis mooma com invasores provenientes do centro e dos lados dos vizinhos menos e mais distantes, e seus respectivos intervalos de confiança.

\section{DISCUSSÃO}

As lutas que demandam maior gasto energético são as que envolvem a conquista e disputa de territórios (WAAGE 1988), espera-se que os custos de interações entre os vizinhos fossem reduzidos em relaçãoaos custos decombatecominvasores(SwITZER \& EASON 2003). A manutenção de territórios que apresentam recursos para reprodução é comum em Odonata (CoNRAD \& Pritchard 1992). Este fenômeno maximiza a probabilidade de sucesso reprodutivo, entretanto, gera custos para manutenção do território (KREBS \& DAVIES 1987). Libélulas apresentam vários comportamentos estilizados relativos à manutenção do território, como por exemplo, interações agonísticas com perseguição e contato corporal (CORBET 1999).

Em espécies territorialistas que defendem recursos limitantes para a reprodução (como no caso de $P$. mooma), há a presença de vários machos que disputam estes territórios, ficando, portanto, agregados. Esta disposição além de refletir a existência de uma hierarquia de dominância nestes territórios (machos que mantém territórios melhores em detrimento de machos com territórios de menor qualidade), há um efeito mais sutil, relativo a disputas territoriais entre machos residentes (mantenedores de áreas) e machos intrusos (machos sem território, que tentam se estabelecer seja por invasão ou por expulsão de um macho residente).

Neste cenário, espera-se que a formação de 'coalizões' entre vizinhos bem estabelecidos ocorra através do reconhecimento entre estes (LOPES \& DE MARCO 2000). Este fenômeno resulta em interações menos agressivas entre os vizinhos e mais agressivas com os intrusos ou invasores (dear enemy effect). Observamos em $P$. mooma que existe um efeito significativo indicando que machos que se reconhecem como vizinhos interagem com menor agressividade do que quando interagem com invasores.

Estas interações com vizinhos sugerem que, apesar de existir um limite claramente definido do raio do território em espécies de Perithemis (Switzer \& EASON 2003), os eventos ligados à invasão de território por vizinhos bem estabelecidos apresentam interações de reconhecimento e patrulha. De MARCo \& RESENDE (2002) observaram que machos de $P$. mooma frequentemente interagem com coespecíficos durante pequenos voos de patrulha em torno de seus territórios. Desta forma, uma vez que os vizinhos estejam estabelecidos, independentemente da distância relativa entre eles, estes se reconhecerão, diluindo o efeito da agressividade como esperado pelo modelo "dear enemy effect".

Para a análise sobre o tempo de briga com invasores provenientes do centro e dos lados dos vizinhos mais e menos distantes, verificou-se que os indivíduos não passam maior tempo 
de interações agonísticas nem brigam mais com invasores provenientes dos lados dos vizinhos mais distantes. Entretanto, ocorreu maior proporção de briga com invasores provenientes dos lados dos vizinhos menos distantes do que com os provenientes do centro.

Assumindo que não exista diferença entre as interações com vizinhos mais distantes e mais próximos (como foi observado neste estudo), o que definiria as interações entre residentes e invasores seria a tentativa de estabelecimento de território pelo invasor (SWitzer \& WALtERS 1999; SWITZER \& EASON 2000; SWITZER 2002). Nestas interações, os machos residentes, durante os voos de transição de poleiros dentro de seu território e/ou voos de patrulha reagiriam agonisticamente contra estes, através de voos de perseguição.

Observou-se que em $P$. mooma existe um efeito significativo indicando que machos que se reconhecem como vizinhos interagem com menor agressividade do que quando interagem com invasores. Estas interações com vizinhos sugerem que, apesar de existir um limite claramente definido do raio do território em espécies de Perithemis, os eventos ligados à invasão de território por vizinhos bem estabelecidos apresentam interações de reconhecimento e patrulha. Desta forma, é possível sugerir que existe efeito "dear enemy" em machos territoriais com territórios próximos em $P$. mooma, visto que houve diferença significativa o no tempo, bem como na intensidade das interações com vizinhos e com invasores. Entretanto, não há diferença entre a intensidade e tempo de interação com vizinhos próximos ou distantes, sugerindo que o efeito não depende da proximidade dos vizinhos e sim do fato de serem vizinhos bem estabelecidos.

\section{AGRADECIMENTOS}

N. Silva-Pinto agradece ao CNPq. Agradecemos aos funcionários da Faculdade de Agronomia da UFG, responsáveis pelos tanques de piscicultura, localizados na Escola de Agronomia e Engenharia de Alimentos da UFG. Além disso, agrademos aos professores Dr. Rogério Pereira Bastos (UFG) e Arthur Ângelo Bispo de Oliveira (UFG) pelo suporte no desenvolvimento do presente trabalho.

\section{REFERÊNCIAS}

Conrad, K.F. \& G. Pritchard, 1992. An ecological classification of odonate mating systems: The relative influence of natural, inter- and intra-sexual selection on males. Biological Journal of the Linnean Society, 45, 255-269.

Corbet, P.S., 1999 Dragonflies: Behavior and Ecology of Odonata. Comstock Publ. Assoc., Ithaca, NY. 830p.

De Marco Jr, P., 1998. The Amazonian Campina dragonfly assemblage: patterns in microhabitat use and behaviour in a foraging habitat (Anisoptera). Odonatologica, 27: 239-248.

De Marco Jr, P. \& D.C. Resende, 2004. Cues for Territory Choice in Two Tropical Dragonflies. Neotropical Entomology, 33, 397-401.
De Marco, P. Jr., 2008. Libellulidae (Insecta: Odonata) from Itapiracó reserve, Maranhão, Brazil: new records and species distribution information. Acta Amazônica, 38: 819-822.

De Marco, P. Jr. \& D.C. Resende, 2002. Activity patterns and thermoregulation in a tropical dragonfly assemblage. Odonatologica, 31: 129-138.

Garrison, R. W., N. Von Ellenrieder \& J. A. Louton, 2010. Damselfly Genera of the New World. Baltimore, an Illustrated and Annotated Key to the Zygoptera. The Johns Hopkins University Press, 528p.

Krebs, J.R. \& N.B. Davies, 1987. An Introduction to Behavioural Ecology. Blackwell Scientific Publications, 420p.

Lopes, F.S. \& P. De Marco, 2000. Comportamento territorial de insetos: aspectos conceituais e estudos de caso. Oecologia Brasiliensis, 8: 193-222.

May, M.L., 1976. Thermoregulation in adaptation to temperature in dragonflies (Odonata: Anisoptera). Ecological Monographs, 46, 1-32.

Marden, J.H. \& Rollins, R.A., 1994. Assessment of energy reserves by damselflies engaged in aerial contests for mating territories. Animal Behaviour, 48, 1023-1030.

Pinto, N.S., 2011. Ocorrência de Orthemis cultriformis (Calvert) (Odonata: Libellulidae) para o Estado de Goiás (Brasil). Entomobrasilis, 4: 36-37.

Switzer, P. \& P.K. Eason, 2003. Space use in territorial amberwing dragonflies: are residents female maximizers or neighbor minimizers? Behavioral Ecology and Sociobiology, 54: 321328.

Switzer, P.V., 2002. Territory quality, habitat selection, and competition in the amberwing dragonfly, Perithemis tenera (Say) (Odonata: Libellulidae): Population patterns as a consequence of individual behavior. Journal of the Kansas Entomological Society, 75: 145-157.

Switzer, P.V. \& P.K. Eason, 2000. Proximate constraints on intruder detection in the dragonfly Perithemis tenera (Odonata: Libellulidae): Effects of angle of approach and background. Annals of the Entomological Society of America, 93: 333-339.

Switzer, P.V. \& W. Walters, 1999. Choice of Lookout Posts by Territorial Amberwing Dragonflies, Perithemis tenera (Anisoptera: Libellulidae). Journal of Insect Behavior, 12, 385-398.

Temeles, E.J., 1994. The role of neighbours in territorial systems: when are they "dear enemies"? Animal Behaviour, 47, 339350 .

Waage, J.K., 1988. Confusion over residency and the escalation of damselfly territorial disputes. Animal Behaviour, 36, 586595 .

Zar,J.H., 1999. Biostatistical Analysis. New Jersey, Prentice-Hall Englewood Cliffs, 663p.

\section{Recebido em: o8/10/2012}

Aceito em: 08/01/2013

\section{Como citar este artigo:}

Pinto, N.S., J. Hidasi Neto, V. Ribeiro, A.R. Rodrigues, B.R. Brandão \& C.O. Rocha, 2013. Efeito da Presença de Vizinhos sobre o Comportamento Territorial de Perithemis mooma (Kirby) (Anisoptera: Libellulidae). EntomoBrasilis, 6(2): 104-107.

Acessível em: http://www.periodico.ebras.bio.br/ojs/index.php/ebras/article/view/285. doi:10.12741/ebrasilis.v6i2.285 